\title{
Computation of Brightness Temperature of Sea-foam Modelled as Sequences of Thin Phase Screens using Matlab
}

\author{
Ayibapreye K. Benjamin, PhD \\ Department of Electrical/Electronics Engineering, \\ Niger Delta University, Bayelsa State, Nigeria \\ Collins E. Ouserigha, PhD \\ Department of Physics, \\ Niger Delta University, Bayelsa State, Nigeria
}

Doi:10.19044/esj.2020.v16n3p425 ～URL:http://dx.doi.org/10.19044/esj.2020.v16n3p425

\begin{abstract}
Sea surface temperature of the ocean is a significant climate parameter. Satellites provide data for analysing and monitoring the sea surface temperature (SST). Satellite remote sensing provide thermal data in a short duration over large area. Temperature measurement by remote sensing is dependent on the principle that most objects emit electromagnetic (EM) radiation corresponding to temperature, wavelength and emissivity of the objects. Brightness temperatures are detected by thermal sensors, however, brightness temperature coincides with the real temperature of objects if they are black bodies. In this paper, we estimated the effective dielectric constant of sea foam layer which is a very important parameter in investigating ocean brightness temperature. This was done at WindSat frequencies and using a discretization method to evaluate the dielectric constant of a random distribution of air-bubbles discretized into slices of sea foam layer. For efficient evaluation of scattering by foam covered sea surface and measurement of brightness temperature in milli-Kelvin, we develop a discrete based physical model of sea foam which provides accurate estimate of the complex effective dielectric constant of sea foam. The foam covered sea foam layer is modelled as sequences of thin phase screens ( slices of sea foam layer) with equal depth . Each layer comprised of random distribution of bubbles that follows a log-normal distribution pattern with geometrical and optical properties such as foam layer thickness, foam void fraction, foam volume fraction, sea surface temperature and sea surface salinity. Results of sea surface emissivity and brightness temperature as a function of polarization, angle of incidence, WindSat frequencies and thickness of sea foam are presented.
\end{abstract}


Keywords: Discrete Method, Brightness Temperature, Sea Foam

\section{Introduction}

Wave breaking influenced by strong wind speed have enormous contribution to microwave radiation and scattering of sea surface. (Pandey and Kakar, 1982), (Wilheit, 1979) proposed various empirical models which were handful to estimate the effects of sea foam on microwave radiation. These were evident as (Smith, 1988; Stogryn, 1972) predicted foam emissivity and brightness temperature fitted as functions of incidence angle and frequency, with experimental data of foam covered sea surface. It was discovered that foam emissivity has strong dependence on foam layer thickness in a waveguide experiment by (Williams, 1971). (Militskii et al., 1978), (Podzimek, 1984) illustrated that foam microstructures and air volume fraction (AVF) are also important factors in estimating the emissivity of sea foam using some empirical emissivity models. Further investigation of the effects of the aforementioned factors on the remote sensing of foam covered sea surface were carried out by (Rose et al., 2002), (Camps et al., 2005) to measure seawater coating thickness, particle size distribution, and the thickness of foam layer. However, it is very difficult for these empirical emissivity models to theoretically disclose how foam layer affects sea surface emission. (Chen et al, 2003), (Huang and Jin, 1995), built theoretical emissivity models such as the vector radiative transfer (VRT) equation and effective medium theory while the dense medium radiative transfer (DMRT) theory have been applied to the sea foam layer (Tsang, et al., 2000), (Guo et al., 2001), (Liu et al., 2006), (Wei and Liu, 2007), (Wei and Ge, 2005), (Lui et al., 2013), (Wei, 2011), (Wei, 2013) for evaluation of sea foam effective dielectric constant. These models achieved some good results but the interactions of air-bubbles coated with seawater are not considered precisely in the sea foam permittivity within a microwave wavelength by a suitable theory. In dense sea foam media, the effective permittivity is a vital parameter of sea foam microwave radiation (Chen et al., 2000). Hence, there is need to propose a more accurate method for estimation of the effective permittivity of sea foam layer.

A new approach of modelling sea foam is proposed to account for the optical properties such as foam layer thickness, foam void fraction, bubble radius, bubble size distribution (BSD), and bubble shape. The bubble size distribution and bubble radius are evaluated by inverse cumulative error function using Newton Raphson method. Three dimensional sphere packing is applied for filling the bubbles in the domain. The domain could be cube, cuboid, prism, or cylinder, and the packing is implemented such that no two adjacent bubbles overlap. The random close packing of spheres is used for the irregular arrangement of spheres so that they are densely packed with the 
bubble volume fraction $64 \%$, while the space within the cube or other domain geometry is $36 \%$, for maximum packing density.

\section{Discrete Method for Evaluation of Sea Foam Effective Dielectric Constant}

The discrete method is implemented by considering 3-dimensional (3D) sphere packing into a 3-D finite domain such as a cube/box. The bubble locations or positions are generated by uniformly distributed random numbers of size $n=1000000$ in $\mathrm{X}, \mathrm{Y}$, and $\mathrm{X}$ coordinates. Bubble radii $r_{i}$ of the $n$ randomly distributed air-bubbles are computed using the inverse method. The inverse method transforms the uniform variate $U(0,1)$ to normal variate with mean $\mu=0$ and variance $\sigma^{2}=1, N(0,1)$. Bubble radii $r_{i}$ and bubble size distribution $N(r)$ were computed assuming a mean $\mu=2.0$ and standard deviation $\sigma=0.5$, geometric mean $\mu_{g}=500 \mu m$ and geometric standard deviation $\sigma_{g}=2.0$, using an empirical expression for computation of the scaled normal variate and bubble size distribution (BSD) which is lognormally distributed.

The 3-D randomly packed spheres in a cubic domain were translated into 2-D slices of solid annuli. The conversion from 3-D to 2-D slices was achieved by calculating the radius of each individual circle based on the concept of intersection of a sphere and a plane. The 2-D slices were discretized with grid sizes $\Delta x$ and $\Delta y$ which leads to intersection of circles bounded in a unit square with some grid points. The grid sizes were sampled such that the edges of the circle circumference which intersects with grid points farther from the inner grids bounded by the circles are negligible. We were able to estimate the effective dielectric constant by calculating the area of the outer and inner circles. The outer circle is seawater while the inner circle contains $80-90 \%$ air, with known effective dielectric constants of air $\varepsilon_{\text {air }}=1.00005+$ $0.0000 i$ and seawater at WindSat frequencies, we were able to estimate effective dielectric constant of the $n$ randomly distributed spheres for 5 slices. 


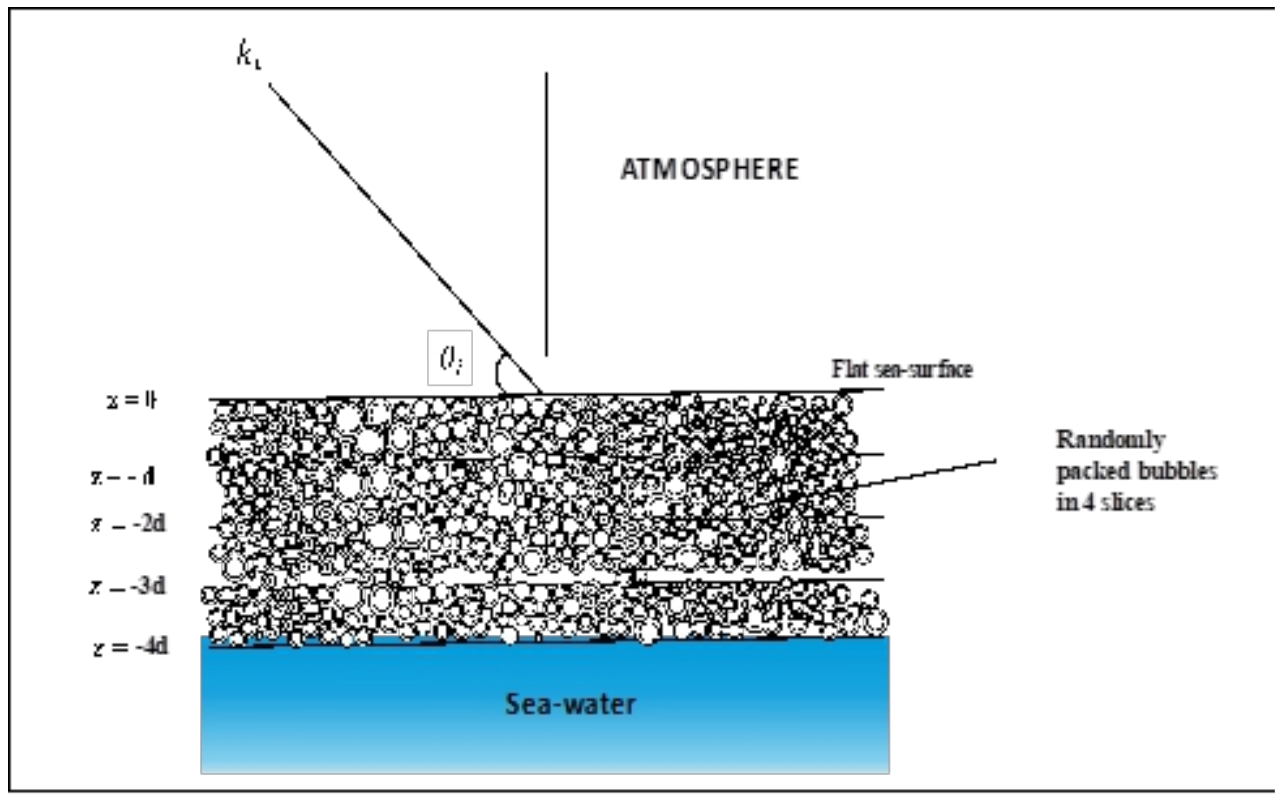

Figure 2.1. Geometrical configuration of foam-covered sea surface shows thermal emission from the flat-sea surface. The foam layer region is divided into 5 slices or layers which are equidistant and accounts for scattering and absorption of millimetre wave ( $\mathrm{mmW})$ at WindSat frequencies.

In this paper, a model that probes the propagation phenomena that occurs when $\mathrm{mmW}$ at frequencies between $10.7 \mathrm{GHz}$ and $37 \mathrm{GHz}$ travels through a sea-foam layer $0 \geq z \geq-d$, where $d$ is the depth of the sea foam layer, is reported. The sea-foam layer is a diphasic composite with numerous isotropic-coated spherical particles, randomly embedded in an isotropic host with permittivity $\varepsilon_{\text {foam }}$ and no overlap between adjacent spherical airbubbles. These air-bubbles consist of core air with permittivity $\varepsilon_{\text {air }}$ and coating shell with permittivity $\varepsilon_{s w}$. For $n$ spherical particles with outer radii $r_{0 N}$ and inner radii $r_{i N}$ randomly distributed in the host medium. The radii are represented by the bubble size distribution of the particles $N(r)$.

The simulation was also implemented for $n=1000000$. The log-normal distribution expressed as

$$
N(r)=\frac{1}{\ln \sigma \cdot r \sqrt{2 \pi}} \times \exp \left\{-0.5\left[\frac{\ln \frac{r}{\bar{r}}}{\ln \sigma_{g}}\right]^{2}\right\}
$$

$N(r)$ was calculated in Fortran adopting a geometric mean deviation $\sigma_{g}=$ 2.0 and mean radius $\bar{r}=500 \mu m$.

Equation (2.1) was compared with a scaled normal distribution with mean $=$ 2.0 and standard deviation $=0.5$. 
$\mathrm{X}=\sigma Z+\mu$, where $\mathrm{X}$ is the scaled normal variate, $\sigma$ the standard deviation and $\mu$ the mean.

We substitute $X=\left\{\left[\frac{\ln \frac{r}{\bar{r}}}{\ln \sigma_{g}}\right]^{2}\right\}$ and was able to evaluate the bubble radii $r$ as $r=\bar{r} \cdot \exp \left(X \times \ln \sigma_{g}\right)$

\section{Estimated Sea Foam Complex Effective Dielectric Constant}

For fixed salinity $(34 \mathrm{psu})$, sea surface temperature $\left(20^{\circ} \mathrm{C}\right)$ and frequency range between $1.4 \mathrm{GHz}$ to $37.0 \mathrm{GHz}$, the dielectric constant of sea water was calculated by adopting existing methods by Stogryn, Guillou, Wentz, English, Klein and Swift (Stogryn et.al, 1997). These calculated dielectric constants were used to estimate the effective permitivity of sea foam. The area of the circles in each slice was calculated using the total number of grid points.

The effective dielectric constants of sea foams at frequencies $10.7 \mathrm{GHz}$ and $37.0 \mathrm{GHz}$ were calculated for 5 slices of randomly packed air-bubbles coated with thin layer of seawater.

\begin{tabular}{|c|c|c|}
\hline FREQUENCY & $10.7 \mathrm{GHZ}$ & $37 \mathrm{GHz}$ \\
\hline Slice 1 & $1.0948-0.1251 \mathrm{i}$ & $1.0006-0.0332 \mathrm{i}$ \\
\hline Slice 2 & $1.1248-0.1507 \mathrm{i}$ & $1.0108-0.0239 \mathrm{i}$ \\
\hline Slice 3 & $1.1622-0.1810 \mathrm{i}$ & $1.0225-0.0344 \mathrm{i}$ \\
\hline Slice 4 & $1.1983-0.2072 \mathrm{i}$ & $1.0315-0.0569 \mathrm{i}$ \\
\hline Slice 5 & $1.2271-0.2277 \mathrm{i}$ & $1.0465-0.0637 \mathrm{i}$ \\
\hline
\end{tabular}

Table 3.1. Results for Dielectric constant of sea foam at frequencies of $10.7 \mathrm{GHz}$ and 37 $\mathrm{GHz}$ for 5 2-D Slices of randomly packed air-bubbles covered with thin-layer of seawater.

The effective dielectric constant of sea foam increases with increase in thickness of foam layer and decreases with increase in frequency as illustrated in Figure 3. The effective dielectric constant of sea foam is used in the computation of Fresnel's reflection coefficients for both horizontal $R_{p}^{\text {foam }}$ and vertical $R_{v}^{\text {foam }}$ polarized fields, at the air-foam interface and the foam-ocean interface. The Fresnel's reflection coefficients are used for the computation of sea surface emissivity $e^{\text {foam }}$ and brightness temperature $T_{B}$ in the radiative transfer equations given below.

$$
\begin{gathered}
e_{p}^{\text {foam }}=1-\left|R_{p}^{\text {foam }}\right|^{2} \\
e_{v}^{\text {foam }}=1-\left|R_{v}^{\text {foam }}\right|^{2}
\end{gathered}
$$

where $e_{p}{ }^{\text {foam }}$ is the sea surface emissivity, $p=h$ (horizontal polarization) and $v=s$ (vertical polarization).

$$
\begin{gathered}
T_{B}=e_{p}{ }^{\text {foam }} T_{s} \\
T_{B}=e_{v}{ }^{\text {foam }} T_{s}
\end{gathered}
$$


where $T_{s}$ denote sea surface temperature. The expressions of reflection coefficients for multi-layered foam structure for both horizontal $R_{p}^{\text {foam }}$ and vertical $R_{v}^{\text {foam }}$ polarized fields are given in (Kong.et al, 2000).

$R_{p}$ is the reflection coefficient of the foam layer which describes the amplitudes of a reflected wave relative to an incident wave and is closely related to the transmission coefficient. For the slices of sea foam layer which exemplifies multi-layered composites each layer having different dielectric constants, there are multiple reflections and transmission in each layer caused by the boundary separating of different regions.

The reflection coefficient $R_{p}$ reflects the effect of all transmission and reflection of an incident wave in the stratified medium and its expression for multi-layers are shown in (Kong.et al, 2000).

\section{Comparison of Simulated Emissivity of Sea Foam with Experimental Results}

Brightnes s temperature $T_{B}$ against Incidence angle at $10.7 \mathrm{GHz}$

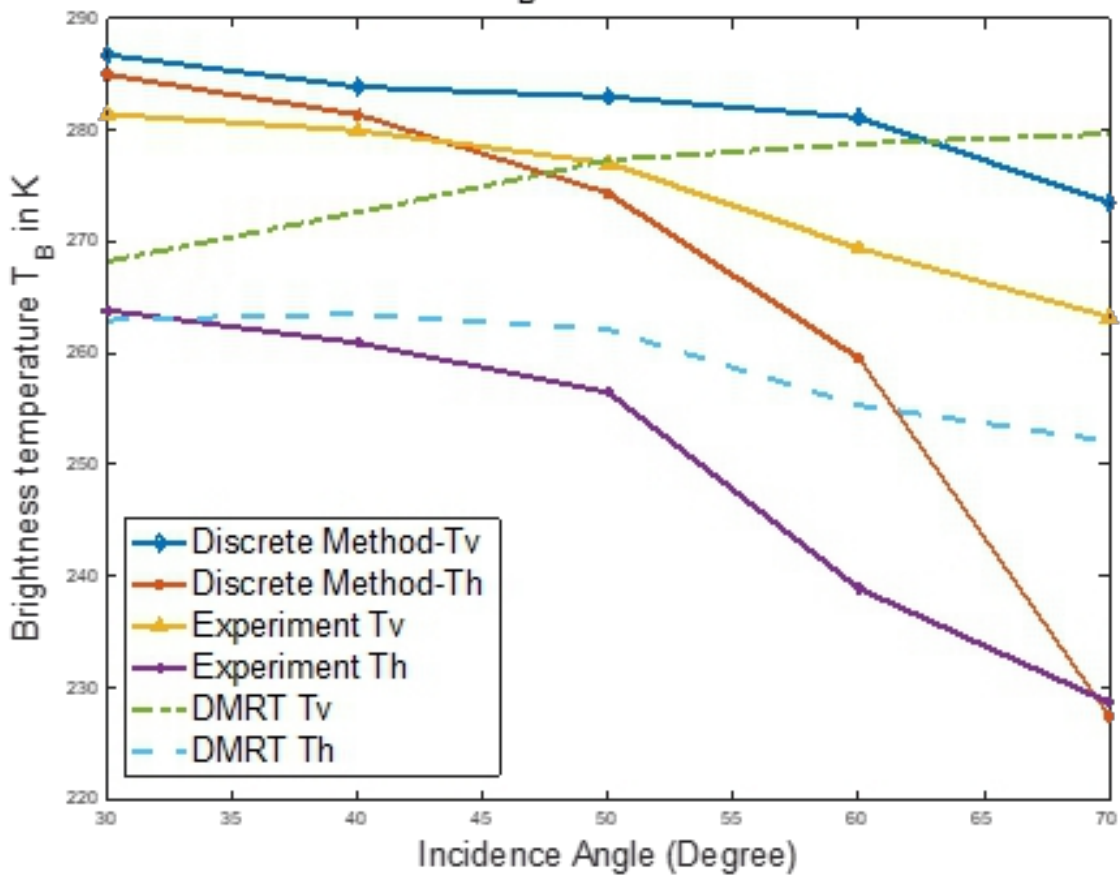

Figure 4.1. Comparison between simulation results and measurements of microwave emissivity at $10.7 \mathrm{GHz}$ for horizontal and vertical polarization as a function of incidence angle.

Figures 4.1 and 4.2 illustrate brightness temperature variation with incidence angles at $10.7 \mathrm{GHz}$ and $37 \mathrm{GHz}$ respectively, for both vertical and horizontal 
polarizations. The brightness temperature as a measure of the radiance of the upward millimetre wave radiation from the sea surface decreases with increase in incident angle. Experimental data used in computation of the brightness temperature, yields brightness temperature that increases at angles $\theta_{i}=30^{\circ}$, $35^{\circ}$ and $40^{\circ}$ then undergoes a decrease at angles $45^{\circ}, 50^{\circ}, 55^{\circ}$ and $60^{\circ}$ for both horizontal and vertical polarizations.

Brightness temperature $\mathrm{T}_{\mathrm{B}}$ against Incidence angle at $37 \mathrm{GHz}$

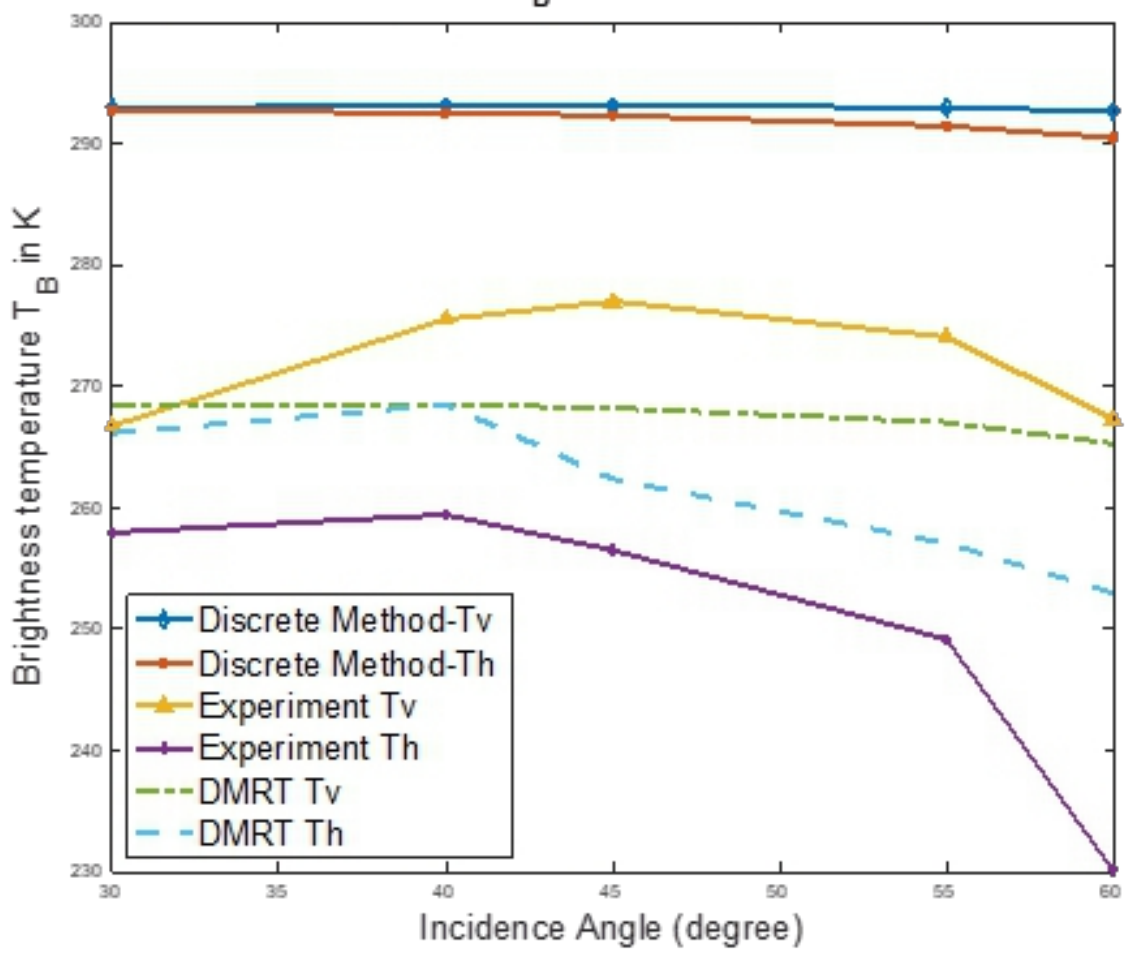

Figure 4.2. Comparison between simulation results and measurements of microwave emissivity at $37 \mathrm{GHz}$ for horizontal and vertical polarization as a function of incidence angle. 


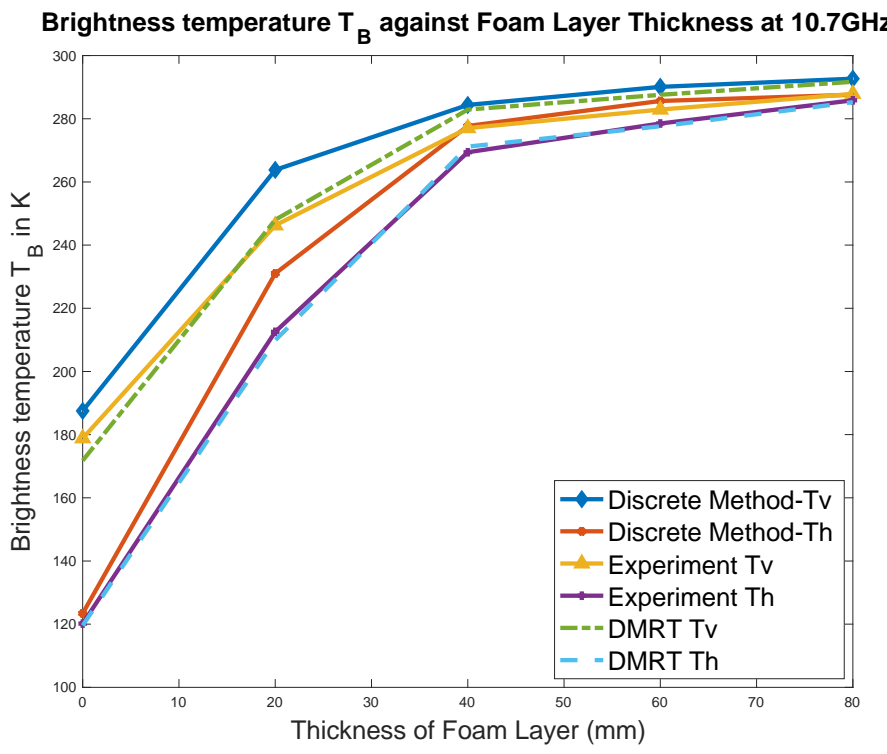

Figure 4.3. Comparison between simulation results and measurements of microwave emissivity at $10.7 \mathrm{GHz}$ for horizontal and vertical polarization as a function of foam layer thickness.

Figures 4.3 and 4.4 show that brightness temperature of the sea surface covered by foam increases with increase in foam layer thickness at frequencies $10.7 \mathrm{GHz}$ and $37 \mathrm{GHz}$. The discrete method used for computation of the brightness temperature of foam covered sea surface, follows similar pattern as experimental and DMRT methods shown in figures 4.3 and 4.4.

The figures show explicitly, that brightness temperature increases as the foam layer thickness increases and later saturates at a specific foam thickness, for both horizontal and vertical polarization. (Chen et.al 2003) DMRT simulation results show that the saturation point of horizontal polarization was slightly larger than that of vertical polarization. The discrete method results show reasonably good agreement with DMRT and experimental measurements. 


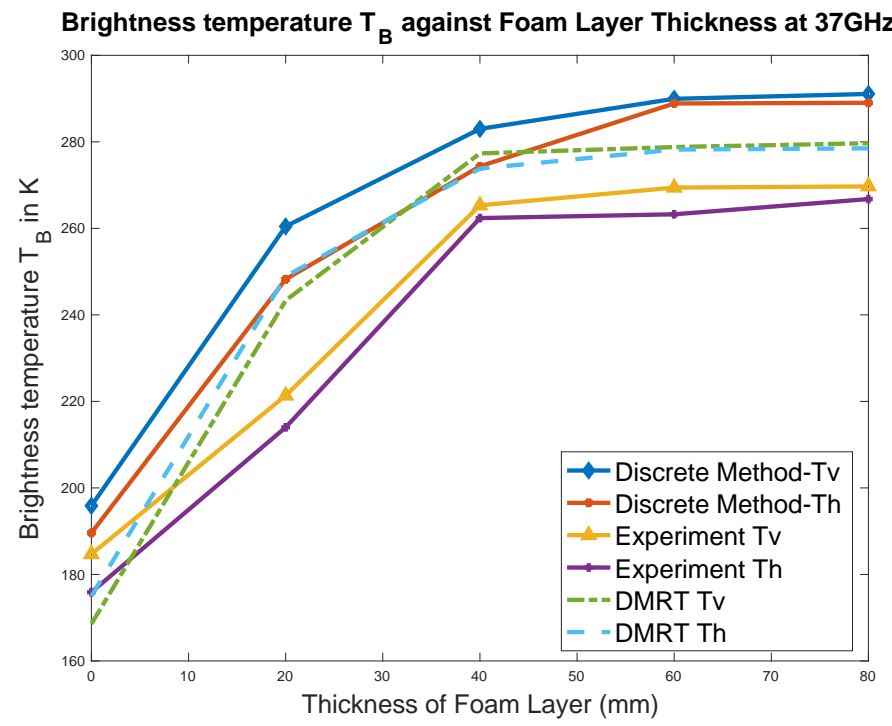

Figure 4.4. Comparison between simulation results and measurements of microwave emissivity at $37 \mathrm{GHz}$ for horizontal and vertical polarization as a function of foam layer thickness.

\section{Conclusion}

Earlier reports suggest that there is negligible scattering due to interaction between EM wave and sea surface covered with foam and absorption been the major contributor to losses in a passive microwave remote sensing. This paper show that with accurate prediction of the effective dielectric constant of sea foam based on its microscopic properties, the scattering properties of the EM wave propagated in a foam-covered sea surface can be investigated. The future investigation of the scattering properties of the sea foam layer, by propagating an incident plane wave through the various 2D slices using the Split-step Fourier method, and comparison of the SSFT results will be compared with Finite-difference method and Pade's approximation of the Split-step method.

\section{References:}

1. Pandey, P.C., and R. K. Kakar, (1982). Remote sensing of the ocean: An empirical microwave emissivity model for a foam-covered sea. 7, no.3, (135-140), IEEE J. Oceanic Eng.

2. Smith, P. M., (1998): Remote sensing of the ocean: The emissivity of sea foam at 19 and $37 \mathrm{GHz}$, , 26, (541-547), IEEE Trans. Geosci. Remote Sensing.

3. Stogryn, A., (1972): Remote sensing of the ocean: The emissivity of sea foam at microwave frequencies, 77, no. 9, (1658-1666). Journal of Geophysical Research. 
4. Wilheit Jr. T.T., (1979): Ocean science: A model for the microwave emissivity of the ocean's surface as a function of wind speed, IEEE Trans. 17, (244-249), Geosci. Electron.

5. Williams, G.F.,(1971): Remote sensing:Microwave emissivity measurements of bubbles and foam. Electron., 9, (221-244) IEEE Trans. Geosci..

6. Militskii, Y. A. et.al. (1978): Atmospheric science: Thermal radio emission from foam structures, Sov. Phy.: (23, 601-602), Engl. Transl.

7. Podzimek, J., (1984): Geosci.: Size spectra of bubbles in the foam patches and sea salt nuclei over the surf zone, , (192-202), Tellus 36 $\mathrm{B}(3)$.

8. Rose, L. A., W. E. Asher, S. C. Reising, P. W. Gaiser, K. M. St Germain, D. J. Dowgiallo, K. A. Horgan, G. Farquharson, and E. Knapp, (2002): Radiometric measurements of the microwave emissivity of foam, Geoscience and Remote Sensing, no. 12, 40, (2619-2625). IEEE Transactions on.

9. Camps, A., M. Vall-Llossera, R. Villarino, N. Reul, B. Chapron, I. Corbella, N. Duffo, F. Torres, J. J. Miranda, R. Sabia, et al., (2005): The emissivity of foam-covered water surface at L-band: Theoretical modelling and experimental results from the frog 2003 field experiment, Geoscience and Remote sensing, , 43, no. 5, (925-937), IEEE Transactions on.

10. Chen, D., L. Tsang, L. Zhou, S. C. Reising, W. E. Asher, L. A. Rose, K.-H. Ding, and C.- T. Chen, (2003): Microwave emission and scattering of foam based on Monte-Carlo simulations of dense media, Geoscience and Remote sensing, 41, no. 4, 782-790, IEEE Transanction on.

11. Huang, X. Z., and Y. Q. Jin, (1995): Scattering and emission from twoscale randomly rough sea surface with foam scatterers Proc. Inst. Elect. Eng., 142,109-114. Microwave Antenna Propagat.

12. Tsang, L., C-T. Chen, A. T. C. Chang, J. Guo, and K. H. Ding, (2000): Dense media radiative transfer based on quasi-crystalline approximation with applications to passive microwave remote sensing of snow, 35, no. 3, 731-749, Radio Sci.

13. Guo, J., L. Tsang, W. Asher, K.-H. Ding, and C.-T. Chen, (2001): Applications of dense media radiative transfer theory for passive microwave remote sensing of foam covered ocean," Geoscience and Remote Sensing, IEEE Transactions on, 39, no. 5, (1019-1027).

14. Lui, S. B., Wei, E. B., Hong, J. L., \& Ge, Y., (2006): Microwave backscattering from the sea surface with breaking waves, Chinese Physics, 15, no. 9, (2175-2179). 
15. Wei, E. B., and Liu, Y., (2007): Application of effective medium approximation theory to ocean remote sensing under wave breaking, Science

16. in China Series D: Earth Science, 50, no. 3, (474-480).

17. Wei, E. B., and Ge, Y., (2005): A microwave emissivity model of sea surface under wave breaking, 34, no. 6, (1259-1264), Chinese Physics.

18. Liu, S., E. Wei, and Y. Jia., (2013): Estimating microwave emissivity of sea foam by Rayleigh method, Journal of Applied Remote Sensing. 7.1, (073598-073598).

19. Wei, E. B., (2011): Microwave vector radiative transfer equation of a sea foam by the second-order Rayleigh approximation, Radio Science, 46, RS5012.

20. Wei, E. B., (2013): Effective medium approximation model of sea foam layer microwave emissivity of a vertical profile, 34 , no. 4, (pp.1180-119) International Journal of Remote Sensing.

21. Chen, C.-T., et al., (2000): Analytical and numerical methods for the scattering by dense media, Geoscience and Remote Sensing Symposium, Proceedings, IGARSS, IEEE International, 5.

22. Kong, J. A., 2000: Electromagnetic Wave Theory. Wiley-Interscience, New York, 720. 